\title{
Luminescent Triarylborane-Functionalized Polystyrene: Synthesis, Photophysical Characterization, and Anion Binding Studies
}

\author{
Kshitij Parab, Krishnan Venkatasubbaiah, and Frieder Jäkle* \\ Department of Chemistry, Rutgers University-Newark, 73 Warren Street, Newark, NJ 07102

\section{Supporting Information}

- Materials and General Methods

- Synthesis and Data of Organoboron Polymers

- Synthesis and Data of Precursors and Model Compounds

- Figure S1.TGA plots of polymers PSBMesBT, PSBMesHBT, and PSBMesNBT.

- Figure S2. (a) Comparison of the absorption spectra of PSBMesHBT, PSBMesNBT, and the respective random copolymer in $\mathrm{CH}_{2} \mathrm{Cl}_{2}$ solution. (b) Comparison of the emission spectra of PSBMesHBT, PSBMesNBT, and the respective random copolymer in $\mathrm{CH}_{2} \mathrm{Cl}_{2}$ solution.

- Figure S3. Determination of fluoride binding constants.

- Figure S4. Comparison of the Stern-Volmer plots for the titration of MBMesHBT and PSBMesHBT.

- Table S1. Calculated orbital energies (eV) from DFT (B3LYP) calculations.

- Table S2. Extended list of calculated electronic transitions from TD-DFT (B3LYP) calculations.

- Molecular Mechanics (MM+) calculations. 


\section{Materials and General Methods.}

2-Bromothiophene, 1-bromohexane, 2-bromomesitylene, $n$-BuLi (1.6M in hexanes), $\left[\mathrm{Bu}_{4} \mathrm{~N}\right] \mathrm{Cl}$, $\left[\mathrm{Bu}_{4} \mathrm{~N}\right] \mathrm{Br}$, and $\left[\mathrm{Bu}_{4} \mathrm{~N}\right] \mathrm{CN}$ were purchased from Acros Organics, $\mathrm{BBr}_{3}(99+\%),\left[\mathrm{Bu}_{4} \mathrm{~N}\right] \mathrm{NO}_{3}$, and $\left[\mathrm{Bu}_{4} \mathrm{~N}\right] \mathrm{F}(1 \mathrm{M}$ in THF) from Aldrich, and trimethyltin chloride (98\%) from Strem Chemicals. $\mathrm{BBr}_{3}$ was further purified by vacuum distillation. Caution! $\mathrm{BBr}_{3}$ is toxic and highly corrosive and should be handled appropriately with great care. Fluorinated grease was used for ground glass joints in all reactions involving boron tribromide. The compounds 5-hexyl-2,2'-bithiophene ${ }^{1}$, and mesitylcopper ${ }^{2}$ were synthesized according to literature procedures. 5-Trimethylstannyl-2,2'bithiophene and 5-hexyl-5'-trimethylstannyl-2,2'-bithiophene were obtained by adaptation of a literature procedure for the synthesis of 5-hexyl-5'-tributylstannyl-2,2'-bithiophene'. Poly(4trimethylsilylstyrene) (PSSi) of $M_{w}=29,200$ and $P D I=1.10$ (GPC-RI) was prepared as previously reported. ${ }^{3}$ All reactions were carried out under inert atmosphere using Schlenk techniques or a glove box (Innovative Technologies). Ether solvents were distilled from $\mathrm{Na} /$ benzophenone prior to use. Hydrocarbon and chlorinated solvents were purified using a solvent purification system (Innovative Technologies; alumina/copper columns for hydrocarbon solvents), and the chlorinated solvents were subsequently distilled from $\mathrm{CaH}_{2}$ and degassed via several freeze-pump-thaw cycles.

Instrumentation. All $499.893 \mathrm{MHz}{ }^{1} \mathrm{H}, 125.7 \mathrm{MHz}{ }^{13} \mathrm{C}, 160.4 \mathrm{MHz}{ }^{11} \mathrm{~B} \mathrm{NMR}$, and $186.4 \mathrm{MHz}$ ${ }^{119} \mathrm{Sn}$ NMR spectra were recorded on a Varian INOVA spectrometer equipped with a boron-free 5 $\mathrm{mm}$ dual broadband gradient probe (Nalorac, Varian Inc., Martinez, CA). Solution ${ }^{1} \mathrm{H}$ and ${ }^{13} \mathrm{C}$ NMR spectra were referenced internally to solvent signals. ${ }^{11} \mathrm{~B}$ NMR spectra were referenced externally to $\mathrm{BF}_{3} \cdot \mathrm{Et}_{2} \mathrm{O}(\delta=0)$ and ${ }^{119} \mathrm{Sn}$ NMR spectra were referenced to $\mathrm{SnMe}_{4}$ in $\mathrm{C}_{6} \mathrm{D}_{6}(\delta=0)$.

${ }^{11} \mathrm{~B}$ NMR spectra were acquired with boron-free quartz NMR tubes. All NMR spectra were recorded at ambient temperature unless noted otherwise. The abbreviations Th (2-thienyl), BT (2,2'-bithiophene), HBT (5-hexyl-2,2'bithiophene), $\mathrm{Ph}$ (phenyl), and Hex ( $n$-hexyl) are used for the peak assignments and the labeling scheme shown here was applied.

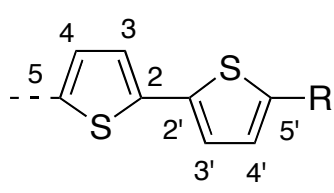

$\mathrm{R}=\mathrm{H}, \mathrm{Hex}, \mathrm{NPh}_{2}$<smiles>CC(C)(C)c1ccc(P)cc1</smiles><smiles>COC(=O)c1cc(C)cc(C)c1P</smiles>

Solution UV-visible measurements were performed in $\mathrm{CH}_{2} \mathrm{Cl}_{2}$ using a Varian Cary 500 scan UV-Vis-NIR spectrophotometer with a $1 \mathrm{~cm}$ quartz cuvette. The fluorescence data were measured on a Varian Cary Eclipse Fluorescence spectrophotometer with the same solutions as those used in the UV-visible measurements. Anthracene was used as the standard for determination of the quantum yields $(\phi)$. The quantum yield of anthracene was adopted from the Handbook of Photochemistry ${ }^{4}$ and the concentration of anthracene in $\mathrm{CH}_{2} \mathrm{Cl}_{2}$ was $6.62 \cdot 10^{-6} \mathrm{M}$. An SCS I G3P-8 Spin Coat System from Specialty Coating Systems Inc. was used to spin cast thin films from toluene on $1 \mathrm{~cm} \times 1 \mathrm{~cm}$ glass slides from Fisher Scientific at $3000 \mathrm{rpm}$. For the titration experiments sample solutions and $\mathrm{Bu}_{4} \mathrm{NF}$ solutions in THF were prepared using a microbalance $( \pm 0.1 \mathrm{mg})$, microliter syringes, and volumetric glassware. The experiments were performed by successive addition of aliquots of a $\mathrm{Bu}_{4} \mathrm{NF}$ solution in THF. Binding constants were determined with the program Hyperquad ${ }^{\mathrm{TM}}$. 5

The DFT calculations have been performed with the Gaussian03 program. ${ }^{6}$ Geometries and electronic properties are calculated by means of hybrid density functional B3LYP with the basis set of $6-31 \mathrm{G}(\mathrm{d})$. The input files and orbital representations were generated with Gaussview (scaling radii of 75\%). Excitation data were calculated using TD-DFT (B3LYP). 
Gel permeation chromatography (GPC) analyses were performed in THF with $0.1 \% \mathrm{Bu}_{4} \mathrm{NBr}^{(1}$ $\mathrm{mL} / \mathrm{min}$ ) using a Waters Breeze system equipped with a 717plus autosampler, a 1525 binary HPLC pump, a 2487 dual $\lambda$ absorbance detector, and a 2414 refractive index detector. A series of styragel columns (Polymer Laboratories; $5 \mu \mathrm{m}$ Mix-D, $5 \mu \mathrm{m}$ Mix-C, and $10 \mu \mathrm{m}$ Mix-B), which were kept in a column heater at $35^{\circ} \mathrm{C}$, were used for separation. The columns were calibrated with PS standards (Polymer Laboratories).

Differential scanning calorimetry (DSC) measurements were performed on a Perkin Elmer Differential Scanning Calorimeter Pyris 1 system with ca. $10 \mathrm{mg}$ of polymer using the specified scan rate. Thermogravimetric analyses (TGA) were performed under $\mathrm{N}_{2}$ atmosphere using a Perkin Elmer Pyris 1 system with ca. $5 \mathrm{mg}$ of polymer at a heating rate of $20{ }^{\circ} \mathrm{C} / \mathrm{min}$ from $50{ }^{\circ} \mathrm{C}$ to $800{ }^{\circ} \mathrm{C}$.

GC-MS spectra were acquired on a Hewlett Packard HP 6890 Series GC system equipped with a series 5973 mass selective detector and a series 7683 injector. A temperature profile with a heating rate of $20^{\circ} \mathrm{C} / \mathrm{min}$ from $50{ }^{\circ} \mathrm{C}$ to $300{ }^{\circ} \mathrm{C}$ was used. Mass spectral data in $+\mathrm{FAB}$ mode with NPOE (2-nitrophenyl octyl ether) as matrix were obtained at the Michigan State University Mass Spectrometry Facility, which is supported, in part, by a grant (DRR-00480) from the Biotechnology Research Technology Program, National Center for Research Resources, National Institutes of Health. Elemental analyses were obtained from Quantitative Technologies Inc. Whitehouse, NJ.

\section{Synthesis and Data of Organoboron Polymers}

Synthesis of PSBMesBT: A solution of $\mathrm{BBr}_{3}(0.60 \mathrm{~g}, 2.39 \mathrm{mmol})$ in $\mathrm{CH}_{2} \mathrm{Cl}_{2}(15 \mathrm{~mL})$ was added dropwise to a solution of poly(4-trimethylsilylstyrene) $\left(0.35 \mathrm{~g}\right.$, ca. $1.98 \mathrm{mmol}$ of $\mathrm{Me}_{3} \mathrm{Si}$ groups $)$ in $\mathrm{CH}_{2} \mathrm{Cl}_{2}(15 \mathrm{~mL})$ and stirred for $14 \mathrm{~h}$. A solution of 2-trimethylstannyl-5,5'-bithiophene $(0.85 \mathrm{~g}$, $2.58 \mathrm{mmol})$ in $\mathrm{CH}_{2} \mathrm{Cl}_{2}(15 \mathrm{~mL})$ was added dropwise at ambient temperature. The reaction mixture turned light yellow and eventually yellow-greenish. The mixture was allowed to stir for $12 \mathrm{~h}$ at room temperature. All volatile components were then removed under high vacuum. The solid was taken up in toluene $(30 \mathrm{~mL})$, and a solution of $\mathrm{MesCu} \cdot\left(\mathrm{C}_{7} \mathrm{H}_{8}\right)_{0.2}(0.53 \mathrm{~g}, 2.64 \mathrm{mmol})$ in toluene $(15 \mathrm{~mL})$ was added dropwise. Formation of a purple precipitate occurred. The reaction mixture was allowed to react for $1 \mathrm{~h}$ at ambient temperature and then refluxed for $24 \mathrm{~h}$. Filtration through celite gave a pale yellow solution that shows blue luminescence. The solution was concentrated to ca. $3 \mathrm{~mL}$ and precipitated into hexanes $(350 \mathrm{~mL})$. The product was purified by repeated precipitation from toluene into hexanes. The precipitate was dried at $50{ }^{\circ} \mathrm{C}$ under high vacuum to obtain a fine light yellow powder $(0.410 \mathrm{~g}, 37 \%)$. For PSBMesBT: ${ }^{11} \mathrm{~B}$ NMR $(160.386 \mathrm{MHz}$, $\left.\mathrm{CDCl}_{3}\right): \delta=50 \mathrm{ppm}\left(w_{1 / 2}=2900 \mathrm{~Hz}\right) ;{ }^{1} \mathrm{H} \mathrm{NMR}\left(499.893 \mathrm{MHz}, \mathrm{CDCl}_{3}\right): \delta=7.8-7.3(\mathrm{br} \mathrm{m}, 4 \mathrm{H}$, $\mathrm{Ph}_{\mathrm{o}}$ and Th-H3,4), 7.1-6.9 (br m, 2H, $\mathrm{Ph}_{\mathrm{m}}$ ), 6.9-6.7 (br m, 1H, Th-H3'), 6.7-6.5 (br m, 4H, ThH4',5' and $\mathrm{Mes}_{\mathrm{m}}$ ), 2.2 (br, 3H, $p$-Me), 1.8 (br, 6H, $o-\mathrm{Me}$ ), the backbone protons are overlapping; ${ }^{13} \mathrm{C}$ NMR $\left(125.69 \mathrm{MHz}, \mathrm{CDCl}_{3}\right): \delta=149.9,143.9,143.7,142.6,139.1,138.8$ (br), 138.5, 137.3, 136.9, 128.1, 127.2 (br), 125.9, 125.2 (aromatic C), 42-40 (polymer backbone), 22.7 (o-Me), 21.4 ( $p$-Me); GPC-RI (THF vs. PS standards): $M_{\mathrm{n}}=26,550, M_{\mathrm{w}}=40,174, P D I=1.51$; UV-Vis $\left(\mathrm{CH}_{2} \mathrm{Cl}_{2}, 3.210^{-5} \mathrm{M}\right): \lambda_{\max }=262 \mathrm{~nm}(\varepsilon=21,992), 368 \mathrm{~nm}(\varepsilon=34,559)$; fluorescence $\left(\mathrm{CH}_{2} \mathrm{Cl}_{2}, 3.2\right.$ $\left.10^{-5} \mathrm{M}\right): \lambda_{\mathrm{em}, \max }=442(\mathrm{sh}), 463 \mathrm{~nm}, \Phi=0.66\left(\lambda_{\mathrm{exc}}=368 \mathrm{~nm}\right) ; \mathrm{UV}-V$ is (thin film): $\lambda_{\max }=292 \mathrm{~nm}$, $373 \mathrm{~nm}$; fluorescence (thin film): $\lambda_{\mathrm{em}, \max }=468,489(\mathrm{sh}) \mathrm{nm}\left(\lambda_{\mathrm{exc}}=373 \mathrm{~nm}\right.$ ); DSC (onset, 20 ${ }^{\circ} \mathrm{C} / \mathrm{min}$; second heating curve $): T_{\mathrm{g}}=165^{\circ} \mathrm{C}$; TGA $\left(20^{\circ} \mathrm{C} / \mathrm{min} ; \mathrm{N}_{2}\right): 51 \%$ weight loss between 248 
${ }^{\circ} \mathrm{C}$ and $339{ }^{\circ} \mathrm{C}$; $15 \%$ weight loss between $381{ }^{\circ} \mathrm{C}$ and $417{ }^{\circ} \mathrm{C}: 9 \%$ residual mass at $800{ }^{\circ} \mathrm{C}$; elemental analysis: calculated C 75.37, H 5.82; found C 75.50, H 6.07.

Synthesis of PSBMesNBT: A solution of $\mathrm{BBr}_{3}$ (ca. $0.44 \mathrm{~g}, 1.76 \mathrm{mmol}$ ) in $\mathrm{CH}_{2} \mathrm{Cl}_{2}(10 \mathrm{~mL}$ ) was added dropwise to a solution of poly(4-trimethylsilylstyrene) $\left(0.255 \mathrm{~g}\right.$, ca. $1.45 \mathrm{mmol}$ of $\mathrm{Me}_{3} \mathrm{Si}$ groups) in $\mathrm{CH}_{2} \mathrm{Cl}_{2}(10 \mathrm{~mL})$, and the mixture was stirred for $16 \mathrm{~h}$. A solution of 5trimethylstannyl-5'-diphenylamino-2,2'-bithiophene (0.93 g, $1.87 \mathrm{mmol})$ in $\mathrm{CH}_{2} \mathrm{Cl}_{2}(15 \mathrm{~mL})$ was added dropwise at RT. The mixture turned dark red and was then allowed to stir for another $12 \mathrm{~h}$. All volatile components were removed under high vacuum. The resulting dark red solid was taken up in toluene $(20 \mathrm{~mL})$, and a solution of $\mathrm{MesCu} \cdot\left(\mathrm{C}_{7} \mathrm{H}_{8}\right)_{0.2}(0.39 \mathrm{~g}, 1.94 \mathrm{mmol})$ in toluene $(10 \mathrm{~mL})$ was added dropwise. Formation of a purple precipitate occurred. The reaction mixture was stirred for $1 \mathrm{~h}$ and then refluxed for $72 \mathrm{~h}$. Filtration through celite gave an orange-red solution, which was concentrated to ca. $3 \mathrm{~mL}$ and precipitated into cold hexanes $(350 \mathrm{~mL})$. The product was purified by repeated precipitation from toluene into cold ether $(350 \mathrm{~mL})$. The solid was dried at $50{ }^{\circ} \mathrm{C}$ under high vacuum to obtain a fine yellow powder $(0.49 \mathrm{~g}$, 60\%). For PSBMesNBT: ${ }^{11} \mathrm{~B}$ NMR $\left(160.386 \mathrm{MHz} \mathrm{CDCl}_{3}\right): \delta=47 \mathrm{ppm}\left(w_{1 / 2}=2000 \mathrm{~Hz}\right) ;{ }^{1} \mathrm{H} \mathrm{NMR}$ $\left(499.893 \mathrm{MHz}, \mathrm{CDCl}_{3}\right.$ ): $\delta=7.37,7.16,7.09,6.97,6.7-6.5,6.5-6.3$ (aromatic H), 2.2 (br, 3H, $p$ $\mathrm{Me}), 1.8$ (br, $6 \mathrm{H}, o-\mathrm{Me})$, the backbone protons are overlapping; ${ }^{13} \mathrm{C} \mathrm{NMR}\left(125.69 \mathrm{MHz}, \mathrm{CDCl}_{3}\right)$ : $\delta=152.5,150.2$ (br), 147.6, 143.3 (br), 142.7 (br), 138.1 (br), 136.9 (br), 129.5, 127.1 (br), 124.8 (br), 124.3 (br), 123.7, 123.3, 120.3 (aromatic C), 42-40 (polymer backbone), 22.8 (o-Me), 21.5 ( $p$-Me); GPC-RI (THF vs. PS standards): $M_{\mathrm{n}}=46,016, M_{\mathrm{w}}=60,412, P D I=1.31$; UV-Vis $\left(\mathrm{CH}_{2} \mathrm{Cl}_{2}, 3.210^{-5} \mathrm{M}\right): \lambda_{\max }=301 \mathrm{~nm}(\varepsilon=27,176), 433 \mathrm{~nm}(\varepsilon=33,930)$; fluorescence $\left(\mathrm{CH}_{2} \mathrm{Cl}_{2}, 3.2\right.$ $\left.10^{-5} \mathrm{M}\right): \lambda_{\mathrm{em}, \max }=537 \mathrm{~nm}, \Phi=0.35\left(\lambda_{\mathrm{exc}}=433 \mathrm{~nm}\right)$; UV-Vis (thin film): $\lambda_{\max }=440 \mathrm{~nm}$; fluorescence (thin film): $\lambda_{\mathrm{em}, \max }=536 \mathrm{~nm}\left(\lambda_{\mathrm{exc}}=440 \mathrm{~nm}\right.$ ); DSC (onset, $20^{\circ} \mathrm{C} / \mathrm{min}$; second heating curve): $T_{\mathrm{g}}=165^{\circ} \mathrm{C}$; TGA $\left(20^{\circ} \mathrm{C} / \mathrm{min} ; \mathrm{N}_{2}\right)$ : $60 \%$ weight loss between $275^{\circ} \mathrm{C}$ and $337{ }^{\circ} \mathrm{C} ; 13 \%$ weight loss between $397{ }^{\circ} \mathrm{C}$ and $436{ }^{\circ} \mathrm{C} ; 8 \%$ residual mass at $800{ }^{\circ} \mathrm{C}$; elemental analysis: calculated C 78.51, H 5.70, N 2.48; found C 76.91, H 5.66, N 2.10.

\section{Synthesis and Data of Precursors and Model Compounds}

Synthesis of 5-Trimethylstannyl-2,2'-bithiophene: A solution of ${ }^{\mathrm{n}} \mathrm{BuLi}$ (113 mL, 1.6M in hexanes, $181 \mathrm{mmol}$ ) was added dropwise over a period of $90 \mathrm{~min}$ to a solution of 2,2'bithiophene $(25 \mathrm{~g}, 150 \mathrm{mmol})$ in THF $(450 \mathrm{~mL})$ at $-78^{\circ} \mathrm{C}$. The mixture was stirred for $25 \mathrm{~min}$ and then allowed to slowly warm up to $0{ }^{\circ} \mathrm{C}$. After cooling the reaction mixture back down to -78 ${ }^{\circ} \mathrm{C}$, a solution of trimethyltin chloride $(36.0 \mathrm{~g}, 181 \mathrm{mmol})$ in THF $(100 \mathrm{~mL})$ was added dropwise. The mixture was stirred at $-78^{\circ} \mathrm{C}$ for $3 \mathrm{~h}$ and then for an additional $12 \mathrm{~h}$ at ambient temperature. Quenching with aqueous $\mathrm{NH}_{4} \mathrm{Cl}$ solution and extraction of the organic layer with ether gave a brown oily material upon evaporation of the solvents. Distillation at $105{ }^{\circ} \mathrm{C}$ under high vacuum $\left(10^{-2}\right.$ torr) gave the product as a colorless liquid $(34.3 \mathrm{~g}, 69 \%) .{ }^{1} \mathrm{H} \mathrm{NMR}\left(499.893 \mathrm{MHz}, \mathrm{CDCl}_{3}\right)$ : $\delta=7.36\left(\mathrm{~d},{ }^{3} \mathrm{~J}=3.5 \mathrm{~Hz}, 1 \mathrm{H}, \mathrm{Th}-\mathrm{H} 3\right), 7.25\left(\mathrm{~m}, 2 \mathrm{H}\right.$, Th-H3,3'), $7.17\left(\mathrm{~d},{ }^{3} \mathrm{~J}=3.5 \mathrm{~Hz}, 1 \mathrm{H}, \mathrm{Th}-\mathrm{H} 4\right)$, $7.06\left(\mathrm{~d} / \mathrm{pst},{ }^{3} \mathrm{~J}=23 / 29.5 \mathrm{~Hz}, 1 \mathrm{H}, \mathrm{Th}-4{ }^{\prime}\right), 0.47\left(\mathrm{~s} / \mathrm{d},{ }^{3} \mathrm{~J}\left({ }^{117 / 119} \mathrm{Sn}, \mathrm{H}\right)=55.5 / 57.5 \mathrm{~Hz}, 9 \mathrm{H}, \mathrm{SnMe}_{3}\right) ;{ }^{13} \mathrm{C}$ NMR (125.69 MHz, $\left.\mathrm{CDCl}_{3}\right): 143.0(\mathrm{Th}), 137.7\left(\mathrm{~s} / \mathrm{d},{ }^{3} \mathrm{~J}\left({ }^{117 / 119} \mathrm{Sn},{ }^{13} \mathrm{C}\right)=5 \mathrm{~Hz}, \mathrm{Th}\right), 137.4(\mathrm{Th})$, $136.0\left(\mathrm{~s} / \mathrm{d},{ }^{4} \mathrm{~J}\left({ }^{117 / 119} \mathrm{Sn},{ }^{13} \mathrm{C}\right)=28 \mathrm{~Hz}, \mathrm{Th}\right), 127.9,125.2\left(\mathrm{~s} / \mathrm{d},{ }^{2} \mathrm{~J}\left({ }^{117 / 119} \mathrm{Sn},{ }^{13} \mathrm{C}\right)=40 \mathrm{~Hz}\right), 124.2,123.7$ $(\mathrm{Th}),-8.0\left(\mathrm{~s} / \mathrm{d},{ }^{2} \mathrm{~J}\left({ }^{117 / 119} \mathrm{Sn},{ }^{13} \mathrm{C}\right)=356 / 373 \mathrm{~Hz}, \mathrm{SnMe}_{3}\right) ;{ }^{119} \mathrm{Sn}$ NMR $\left(186.413 \mathrm{MHz}, \mathrm{CDCl}_{3}\right): \delta=$ $-25.2 \mathrm{ppm}$; GC-MS ( $t=12.7 \mathrm{~min}): \mathrm{m} / z$ (\%): $330\left[\mathrm{M}^{+}\right](32), 315\left[\mathrm{M}^{+}-\mathrm{CH}_{3}\right](100)$.

Synthesis of 5-Trimethylstannyl-5'-hexyl-2,2'-bithiophene: A solution of ${ }^{\mathrm{n}} \mathrm{BuLi}(15.3 \mathrm{~mL}$, $1.6 \mathrm{M}$ in hexanes, $24.5 \mathrm{mmol}$ ) was added dropwise over a period of $30 \mathrm{~min}$ to a solution of 2hexyl-5,5'-bithiophene $(5.10 \mathrm{~g}, 20.4 \mathrm{mmol})$ in THF $(150 \mathrm{~mL})$. The reaction mixture was stirred at 
$0{ }^{\circ} \mathrm{C}$ for two hours and cooled down to $-78{ }^{\circ} \mathrm{C}$. The solution was stirred for $12 \mathrm{~h}$. All volatile components were removed under high vacuum, and the residue was taken up in toluene (15 $\mathrm{mL})$. A solution of trimethyltin chloride $(5.20 \mathrm{~g}, 26.1 \mathrm{mmol})$ in THF was then added slowly. The mixture was first stirred at $-78^{\circ} \mathrm{C}$ for $3 \mathrm{~h}$ and then for an additional $12 \mathrm{~h}$ at ambient temperature. Quenching with aqueous $\mathrm{NH}_{4} \mathrm{Cl}$ solution and extraction of the organic layer with ether gave a black oily material upon evaporation of the solvents. Distillation at $140{ }^{\circ} \mathrm{C}$ under high vacuum $\left(10^{-2}\right.$ torr) gave the product as a colorless liquid $(5.10 \mathrm{~g}, 60 \%) .{ }^{1} \mathrm{H} \mathrm{NMR}\left(499.893 \mathrm{MHz}, \mathrm{CDCl}_{3}\right)$ : $\delta=7.21\left(\mathrm{~d} / \mathrm{dd},{ }^{3} \mathrm{~J}=3.5 \mathrm{~Hz},{ }^{4} \mathrm{~J}\left({ }^{117 / 119} \mathrm{Sn}, \mathrm{H}\right)=5.0 \mathrm{~Hz}, 1 \mathrm{H}, \mathrm{Th}-\mathrm{H} 3\right), 7.07\left(\mathrm{~d} / \mathrm{dd},{ }^{3} \mathrm{~J}=3.5 \mathrm{~Hz}\right.$, ${ }^{3} \mathrm{~J}\left({ }^{117 / 119} \mathrm{Sn}, \mathrm{H}\right)=23 / 29.5 \mathrm{~Hz}, 1 \mathrm{H}$, Th-H4), $6.98\left(\mathrm{~d},{ }^{3} \mathrm{~J}=3.5 \mathrm{~Hz}, 1 \mathrm{H}\right.$, Th-H3'), $6.68\left(\mathrm{~d},{ }^{3} \mathrm{~J}=3.5 \mathrm{~Hz}\right.$, 1H, Th-H4'), 2.79 (t, $\left.{ }^{3} \mathrm{~J}=7.0 \mathrm{~Hz}, 2 \mathrm{H}, \mathrm{Hex}\right), 1.69$ (m, 2H, Hex), 1.4-1.3 (m, 6H, Hex), 0.90 (t, 3H, Hex $), 0.44\left(\mathrm{~s} / \mathrm{d},{ }^{3} \mathrm{~J}\left({ }^{117 / 119} \mathrm{Sn}, \mathrm{H}\right)=55.5 / 57.5 \mathrm{~Hz}, 9 \mathrm{H}, \mathrm{SnMe}_{3}\right) ;{ }^{13} \mathrm{C} \mathrm{NMR}\left(125.69 \mathrm{MHz}, \mathrm{CDCl}_{3}\right): \delta=$ 145.2, 143.7, 136.5 (Th), $135.9\left(\mathrm{~s} / \mathrm{d},{ }^{4} \mathrm{~J}\left({ }^{117 / 119} \mathrm{Sn},{ }^{13} \mathrm{C}\right)=27 \mathrm{~Hz}\right.$, Th-C3), 135.0 (Th), 124.8 (Th), $124.4\left(\mathrm{~s} / \mathrm{d},{ }^{3} \mathrm{~J}\left({ }^{117 / 119} \mathrm{Sn},{ }^{13} \mathrm{C}\right)=40 \mathrm{~Hz}\right.$, Th-C4), $123.3(\mathrm{Th}), 31.8,31.7,30.3,28.9,22.8,14.3$ (Hex), $-8.0\left(\mathrm{~s} / \mathrm{d},{ }^{1} \mathrm{~J}\left({ }^{117 / 119} \mathrm{Sn},{ }^{13} \mathrm{C}\right)=356 / 373 \mathrm{~Hz}, \mathrm{SnMe}_{3}\right) ;{ }^{119} \mathrm{Sn} \mathrm{NMR}\left(186.413 \mathrm{MHz}, \mathrm{CDCl}_{3}\right): \delta=-25.6$ ppm; GC-MS ( $t=11.79 \mathrm{~min}): \mathrm{m} / z$ (\%): $414\left[\mathrm{M}^{+}\right](40), 399\left[\mathrm{M}^{+}-\mathrm{CH}_{3}\right](100)$.

Synthesis of 5-Trimethylstannyl-5'-diphenylamino-2,2'-bithiophene: A solution of ${ }^{\mathrm{n}} \mathrm{BuLi}$ (16.5 mL, 1.6M in hexanes, $26.4 \mathrm{mmol}$ ) was added at $-78^{\circ} \mathrm{C}$ over a period of $1 \mathrm{~h}$ to a solution of 5-diphenylamino-2,2'-bithiophene $(7.30 \mathrm{~g}, 21.9 \mathrm{mmol})$ in THF $(250 \mathrm{~mL})$. The color gradually changed from yellow to green. The reaction mixture was stirred at $-78{ }^{\circ} \mathrm{C}$ for 20 min and slowly allowed to warm up to ambient temperature. After stirring for $3 \mathrm{~h}$, the mixture was once again cooled to $-78{ }^{\circ} \mathrm{C}$ and treated with a solution of $\mathrm{Me}_{3} \mathrm{SnCl}(5.70 \mathrm{~g}, 28.6 \mathrm{mmol})$ in THF (100 mL). The reaction solution was allowed to warm to room temperature and stirred for $12 \mathrm{~h}$. Standard aqueous workup provided a black oily material, which was crystallized from hot methanol to give a dark green solid (5.50 g, 50\%). ${ }^{1} \mathrm{H}$ NMR (499.893 MHz, $\left.\mathrm{CDCl}_{3}\right): 7.28\left(\mathrm{~m}, 4 \mathrm{H}, \mathrm{NPh}_{\mathrm{m}}\right), 7.19(\mathrm{~m}$, $4 \mathrm{H}, \mathrm{NPh}_{\mathrm{o}}$ ), $7.17\left(\mathrm{~d},{ }^{3} \mathrm{~J}=4.0 \mathrm{~Hz}, 1 \mathrm{H}, \mathrm{Th}-\mathrm{H} 4\right), 7.06\left(\mathrm{~d},{ }^{3} \mathrm{~J}=4.0 \mathrm{~Hz}, 1 \mathrm{H}, \mathrm{Th}-\mathrm{H} 3\right), 7.05(\mathrm{~m}, 2 \mathrm{H}$, $\left.\mathrm{NPh}_{\mathrm{p}}\right), 6.96\left(\mathrm{~d},{ }^{3} \mathrm{~J}=4.0 \mathrm{~Hz}, 1 \mathrm{H}, \mathrm{Th}-\mathrm{H} 3{ }^{\prime}\right), 6.61\left(\mathrm{~d},{ }^{3} \mathrm{~J}=4.0 \mathrm{~Hz}, 1 \mathrm{H}, \mathrm{Th}-\mathrm{H} 4{ }^{\prime}\right), 0.38(\mathrm{~s} / \mathrm{d}$, $\left.{ }^{3} \mathrm{~J}\left({ }^{117 / 119} \mathrm{Sn}, \mathrm{H}\right)=55.5 / 57.5 \mathrm{~Hz}, 9 \mathrm{H}, \mathrm{SnMe}_{3}\right) ;{ }^{13} \mathrm{C} \mathrm{NMR}\left(125.69 \mathrm{MHz}, \mathrm{CDCl}_{3}\right): \delta=150.4(\mathrm{Th})$, $147.9\left(\mathrm{NPh}_{\mathrm{i}}\right), 143.6(\mathrm{Th}), 136.9(\mathrm{Th}), 135.9\left(\mathrm{~s} / \mathrm{d},{ }^{4} \mathrm{~J}\left({ }^{117 / 119} \mathrm{Sn},{ }^{13} \mathrm{C}\right)=28 \mathrm{~Hz}, \mathrm{Th}-\mathrm{C} 4\right), 131.9(\mathrm{Th}-\mathrm{C} 8)$, 129.4 $\left(\mathrm{NPh}_{\mathrm{m}}\right), 124.3\left(\mathrm{~s} / \mathrm{d},{ }^{3} \mathrm{~J}\left({ }^{117 / 119} \mathrm{Sn},{ }^{13} \mathrm{C}\right)=40.5 \mathrm{~Hz}, \mathrm{Th}-\mathrm{C} 3\right), 123.3\left(\mathrm{NPh}_{\mathrm{p}}\right), 122.8\left(\mathrm{NPh}_{\mathrm{o}}\right), 122.2$, $121.6(\mathrm{Th}),-8.0\left(\mathrm{~s} / \mathrm{d},{ }^{1} \mathrm{~J}\left({ }^{117 / 119} \mathrm{Sn},{ }^{13} \mathrm{C}\right)=356 / 373 \mathrm{~Hz}, \mathrm{SnMe}_{3}\right) ;{ }^{119} \mathrm{Sn}$ NMR $\left(186.413 \mathrm{MHz}, \mathrm{CDCl}_{3}\right)$ : $\delta=-25.6 \mathrm{ppm}$; GC-MS (t=23.86 min): $\mathrm{m} / z(\%): 497\left[\mathrm{M}^{+}\right](100), 482\left[\mathrm{M}^{+}-\mathrm{CH}_{3}\right](7)$.

Synthesis of MBMesBT: A solution of 5-trimethylstannyl-2,2'-bithiophene (0.38 g, 1.15 $\mathrm{mmol})$ in toluene $(10 \mathrm{~mL})$ was added dropwise to a colorless solution of ${ }^{\mathrm{t}} \mathrm{BuPhBBr}_{2}(0.35 \mathrm{~g}, 1.15$ $\mathrm{mmol})$ in toluene $(10 \mathrm{~mL})$. The resulting solution turned yellow upon complete addition. The reaction mixture was stirred for $12 \mathrm{~h}$ at ambient temperature. All volatile materials were removed under high vacuum and the resulting yellow solid was taken up in toluene $(15 \mathrm{~mL})$. A solution of $\mathrm{MesCu} \bullet\left(\mathrm{C}_{7} \mathrm{H}_{8}\right)_{0.2}(0.24 \mathrm{~g}, 1.19 \mathrm{mmol})$ in toluene $(5 \mathrm{~mL})$ was added slowly, which resulted in the formation of a purple precipitate. The reaction mixture was refluxed for $14 \mathrm{~h}$ and then stirred at room temperature for $2 \mathrm{~h}$ before being filtered through celite. The solvent was removed under high vacuum. Purification by repeated extraction with $\mathrm{CH}_{3} \mathrm{CN}$ and crystallization from $\mathrm{CH}_{3} \mathrm{CN}$ gave the product in the form of light yellow crystals $(0.238 \mathrm{~g}, 55 \%)$. For MBMesBT: ${ }^{11} \mathrm{~B}$ NMR $\left(160.386 \mathrm{MHz}, \mathrm{CDCl}_{3}\right): \delta=62 \mathrm{ppm}\left(w_{1 / 2}=1450 \mathrm{~Hz}\right) ;{ }^{1} \mathrm{H} \mathrm{NMR}\left(499.893 \mathrm{MHz}, \mathrm{CDCl}_{3}\right): \delta=7.87$ $\left(\mathrm{d},{ }^{3} \mathrm{~J}=8.5 \mathrm{~Hz}, 2 \mathrm{H}, \mathrm{Ph}_{\mathrm{o}}\right), 7.67\left(\mathrm{~d},{ }^{3} \mathrm{~J}=4.0 \mathrm{~Hz}, 1 \mathrm{H}, \mathrm{Th}-\mathrm{H} 4\right), 7.48\left(\mathrm{~d},{ }^{3} \mathrm{~J}=8.5 \mathrm{~Hz}, 2 \mathrm{H}, \mathrm{Ph}_{\mathrm{m}}\right), 7.38(\mathrm{~d}$, ${ }^{3} \mathrm{~J}=4.0 \mathrm{~Hz}, 1 \mathrm{H}$, Th-H3), $7.34\left(\mathrm{dd},{ }^{3} \mathrm{~J}=3.5 \mathrm{~Hz},{ }^{4} \mathrm{~J}=1.5 \mathrm{~Hz}, 1 \mathrm{H}, \mathrm{Th}-\mathrm{H} 3\right.$ '), 7.30 (dd, ${ }^{3} \mathrm{~J}=5.0 \mathrm{~Hz},{ }^{4} \mathrm{~J}$ $=1.0 \mathrm{~Hz}, 1 \mathrm{H}$, Th-H5'), 7.05 (dd, $\left.{ }^{3} \mathrm{~J}=5.0 \mathrm{~Hz},{ }^{3} \mathrm{~J}=4.0 \mathrm{~Hz}, 1 \mathrm{H}, \mathrm{Th}-\mathrm{H} 4{ }^{\prime}\right), 6.88$ (s, 2H, Mes $\left.{ }_{\mathrm{m}}\right), 2.37$ (s, 3H, p-Me), 2.07 (s, 6H, o-Me), 1.37 (s, 9H, $\left.\mathrm{CMe}_{3}\right) ;{ }^{13} \mathrm{C} \mathrm{NMR}\left(125.69 \mathrm{MHz}, \mathrm{CDCl}_{3}\right.$ ): $\delta=155.0$ 
$\left(\mathrm{Ph}_{\mathrm{p}}\right), 150.0$ (Th), $143.6(\mathrm{Th}), 138.3\left(\mathrm{Mes}_{\mathrm{o}}\right), 137.4(\mathrm{Th}), 137.3\left(\mathrm{Ph}_{\mathrm{o}}\right), 137.2\left(\mathrm{Mes}_{\mathrm{p}}\right), 128.3(\mathrm{Th})$, $127.2\left(\mathrm{Mes}_{\mathrm{m}}\right), 126.1(\mathrm{Th}), 126.0(\mathrm{Th}), 125.4(\mathrm{Th}), 125.3\left(\mathrm{Ph}_{\mathrm{m}}\right), 35.2\left(\mathrm{CMe}_{3}\right), 31.4\left(\mathrm{CMe}_{3}\right), 22.9$ $(o-\mathrm{Me}), 21.5(p-\mathrm{Me})$; UV-Vis $\left(\mathrm{CH}_{2} \mathrm{Cl}_{2}, 3.210^{-6} \mathrm{M}\right): \lambda_{\max }=280 \mathrm{~nm}(\varepsilon=19,960), 367 \mathrm{~nm}(\varepsilon=$ $34,560)$; fluorescence $\left(\mathrm{CH}_{2} \mathrm{Cl}_{2}, 3.210^{-5} \mathrm{M}\right): \lambda_{\mathrm{em}, \max }=425 \mathrm{~nm}, \Phi=0.72\left(\lambda_{\mathrm{exc}}=367 \mathrm{~nm}\right) ;$ FAB-MS $\mathrm{m} / z(\%): 428.2\left[\mathrm{M}^{+}\right](100), 413.1\left[\mathrm{M}^{+}-\mathrm{CH}_{3}\right](6)$; elemental analysis: calculated C 75.69, H 6.82; found C 75.96, H 7.10.

Synthesis of MBMesHBT: A solution of 5-trimethylstannyl-5'-hexyl-2,2'-bithiophene (0.68 g, $1.65 \mathrm{mmol})$ in toluene $(10 \mathrm{~mL})$ was added dropwise to a colorless solution of ${ }^{\mathrm{t}} \mathrm{BuPhBBr}_{2}(0.500$ $\mathrm{g}, 1.65 \mathrm{mmol})$ in toluene $(10 \mathrm{~mL})$. A solution of $\mathrm{MesCu} \bullet\left(\mathrm{C}_{7} \mathrm{H}_{8}\right)_{0.2}(0.33 \mathrm{~g}, 1.64 \mathrm{mmol})$ in toluene $(5 \mathrm{~mL})$ was then added slowly, which resulted in the formation of a purple precipitate. The reaction mixture was refluxed for $12 \mathrm{~h}$, stirred at room temperature for $2 \mathrm{~h}$, and then filtered through celite. The solvent was removed from the resulting light yellow solution under high vacuum. Purification by repeated extraction with $\mathrm{CH}_{3} \mathrm{CN}$ and crystallization from $\mathrm{CH}_{3} \mathrm{CN}$ gave the product in the form of yellow crystals $(0.40 \mathrm{~g}, 48 \%)$. For MBMesHBT: ${ }^{11} \mathrm{~B}$ NMR $(160.386$ $\left.\mathrm{MHz}, \mathrm{CDCl}_{3}\right): \delta=61 \mathrm{ppm}\left(w_{1 / 2}=1900 \mathrm{~Hz}\right) ;{ }^{1} \mathrm{H}$ NMR $\left(499.893 \mathrm{MHz}, \mathrm{CDCl}_{3}\right): \delta=7.88\left(\mathrm{~d},{ }^{3} \mathrm{~J}=\right.$ $\left.8.5 \mathrm{~Hz}, 2 \mathrm{H}, \mathrm{Ph}_{\mathrm{o}}\right), 7.66\left(\mathrm{~d},{ }^{3} \mathrm{~J}=3.5 \mathrm{~Hz}, 1 \mathrm{H}, \mathrm{Th}-\mathrm{H} 4\right), 7.49\left(\mathrm{~d},{ }^{3} \mathrm{~J}=8.5 \mathrm{~Hz}, 2 \mathrm{H}, \mathrm{Ph}_{\mathrm{m}}\right), 7.31\left(\mathrm{~d},{ }^{3} \mathrm{~J}=3.0\right.$ $\mathrm{Hz}, 1 \mathrm{H}, \mathrm{Th}-\mathrm{H} 3), 7.17$ (d, ${ }^{3} \mathrm{~J}=3.5 \mathrm{~Hz}, 1 \mathrm{H}, \mathrm{Th}-\mathrm{H} 3$ '), $6.89\left(\mathrm{~s}, 2 \mathrm{H}, \mathrm{Mes}_{\mathrm{m}}\right), 6.73\left(\mathrm{~d},{ }^{3} \mathrm{~J}=3.5 \mathrm{~Hz}, 1 \mathrm{H}\right.$, Th-H4'), 2.82 (t, $\left.{ }^{3} \mathrm{~J}=7.0 \mathrm{~Hz}, 2 \mathrm{H}, \mathrm{Hex}\right), 2.39$ (s, 3H, $\left.p-\mathrm{Me}\right), 2.08$ (s, 6H, $\left.o-\mathrm{Me}\right) 1.71(\mathrm{~m}, 2 \mathrm{H}$, Hex), 1.4-1.3 (m, 6H, Hex), 1.39 (s, 9H, CMe $), 0.92$ (t, $\left.{ }^{3} \mathrm{~J}=7.0 \mathrm{~Hz}, 3 \mathrm{H}, \mathrm{Hex}\right) ;{ }^{13} \mathrm{C}$ NMR (125.69 $\left.\mathrm{MHz}, \mathrm{CDCl}_{3}\right): \delta=155.5\left(\mathrm{Ph}_{\mathrm{p}}\right), 150.7,147.4,143.8,143.6(\mathrm{Th}), 143.4\left(\mathrm{Mes}_{\mathrm{i}}\right), 138.3\left(\mathrm{Mes}_{\mathrm{o}}\right), 137.3$ $\left(\mathrm{Ph}_{\mathrm{o}}\right)$, $137.1\left(\mathrm{Mes}_{\mathrm{p}}\right), 134.8(\mathrm{Th}), 127.2\left(\mathrm{Mes}_{\mathrm{m}}\right), 125.4,125.3,125.1(\mathrm{Th}), 125.2\left(\mathrm{Ph}_{\mathrm{m}}\right), 35.2$ $\left(\mathrm{CMe}_{3}\right), 31.8,31.7$ (Hex), $31.4\left(\mathrm{CM} \mathrm{e}_{3}\right), 30.5,28.9$ (Hex), 22.9 (o-Me), 22.8 (Hex), 21.5 (p-Me), 14.3 (Hex); UV-Vis $\left(\mathrm{CH}_{2} \mathrm{Cl}_{2}, 3.210^{-6} \mathrm{M}\right): \lambda_{\max }=273 \mathrm{~nm}(\varepsilon=18,780), 380 \mathrm{~nm}(\varepsilon=41,150)$; fluorescence $\left(\mathrm{CH}_{2} \mathrm{Cl}_{2}, 3.210^{-5} \mathrm{M}\right): \lambda_{\text {em,max }}=437 \mathrm{~nm}, \Phi=0.76\left(\lambda_{\text {exc }}=380 \mathrm{~nm}\right) ;$ FAB-MS $\mathrm{m} / z(\%)$ : $512.3\left[\mathrm{M}^{+}\right](100), 469.3\left[\mathrm{M}^{+}-\mathrm{CH}_{3}\right]$ (11); elemental analysis: calculated $\mathrm{C} 77.32, \mathrm{H}$ 8.06; found: $\mathrm{C}$ 77.28, H 8.09.

Synthesis of MBMesNBT: A solution of 5-trimethylstannyl-5'-diphenylamino-2,2'bithiophene $(0.51 \mathrm{~g}, 1.02 \mathrm{mmol})$ in toluene $(5 \mathrm{~mL})$ was added dropwise to a solution of ${ }^{t} \mathrm{BuPhBBr}_{2}(0.300 \mathrm{~g}, 0.99 \mathrm{mmol})$ in toluene $(5 \mathrm{~mL})$. The color of the reaction mixture turned to orange-red with greenish-yellow fluorescence upon complete addition. After stirring for $12 \mathrm{~h}$, all volatile components were removed under high vacuum. The resulting orange-red solid was taken up in toluene $(10 \mathrm{~mL})$, and a solution of $\operatorname{MesCu}\left(\mathrm{C}_{7} \mathrm{H}_{8}\right)_{0.2}(0.21 \mathrm{~g}, 1.04 \mathrm{mmol})$ in toluene $(5 \mathrm{~mL})$ was added slowly, which resulted in the formation of a purple precipitate. The reaction mixture was refluxed for $12 \mathrm{~h}$ and then stirred at room temperature for $2 \mathrm{~h}$. The mixture was filtered through celite and the solvent was removed under high vacuum. Subsequent recrystallization from $\mathrm{CH}_{3} \mathrm{CN}$ gave the product as a yellow powder $(0.23 \mathrm{~g}, 39 \%)$. For MBMesNBT: ${ }^{11} \mathrm{~B}$ NMR $\left(160.386 \mathrm{MHz}, \mathrm{CDCl}_{3}\right): \delta=61 \mathrm{ppm}\left(w_{1 / 2}=1600 \mathrm{~Hz}\right) ;{ }^{1} \mathrm{H} \mathrm{NMR}\left(499.893 \mathrm{MHz}, \mathrm{CDCl}_{3}\right): \delta=7.86$ $\left(\mathrm{d},{ }^{3} \mathrm{~J}=8.5 \mathrm{~Hz}, 2 \mathrm{H}, \mathrm{BPh}_{\mathrm{o}}\right), 7.63\left(\mathrm{~d},{ }^{3} \mathrm{~J}=3.5 \mathrm{~Hz}, 1 \mathrm{H}, \mathrm{Th}-\mathrm{H} 4\right), 7.48\left(\mathrm{~d},{ }^{3} \mathrm{~J}=8.5 \mathrm{~Hz}, 2 \mathrm{H}, \mathrm{BPh}_{\mathrm{m}}\right), 7.30$ $\left(\mathrm{m}, 4 \mathrm{H}, \mathrm{NPh}_{\mathrm{m}}\right), 7.23\left(\mathrm{~d},{ }^{3} \mathrm{~J}=4.0 \mathrm{~Hz}, 1 \mathrm{H}, \mathrm{Th}-\mathrm{H} 3\right), 7.21\left(\mathrm{~m}, 4 \mathrm{H}, \mathrm{NPh}_{\mathrm{o}}\right), 7.13\left(\mathrm{~d},{ }^{3} \mathrm{~J}=4.0 \mathrm{~Hz}, 1 \mathrm{H}\right.$, Th-H3'), 7.09 (m, 2H, $\mathrm{NPh}_{\mathrm{p}}$ ), 6.88 (s, 2H, $\mathrm{Mes}_{\mathrm{m}}$ ), 6.59 (d, ${ }^{3} \mathrm{~J}=4.0 \mathrm{~Hz}, 1 \mathrm{H}, \mathrm{Th}-\mathrm{H} 4$ '), 2.37 (s, 3H, $p$-Me), 2.07 (s, 6H, $o$-Me) $1.37\left(\mathrm{~s}, 9 \mathrm{H}, \mathrm{CMe}_{3}\right) ;{ }^{13} \mathrm{C} \mathrm{NMR}\left(125.69 \mathrm{MHz}, \mathrm{CDCl}_{3}\right): \delta=155.5\left(\mathrm{Ph}_{\mathrm{p}}\right)$, $152.8(\mathrm{Th}), 150.7(\mathrm{Th}), 147.7\left(\mathrm{Nph}_{\mathrm{i}}\right), 143.8(\mathrm{Th}), 143.4\left(\mathrm{Mes}_{\mathrm{i}}\right), 142.9\left(\mathrm{Ph}_{\mathrm{i}}\right), 138.3\left(\mathrm{Mes}_{\mathrm{o}}\right), 137.2$ $\left(\mathrm{Ph}_{\mathrm{o}}\right), 137.1\left(\mathrm{Mes}_{\mathrm{p}}\right), 129.5\left(\mathrm{NPh}_{\mathrm{m}}\right), 127.2\left(\mathrm{Mes}_{\mathrm{m}}\right), 125.2\left(\mathrm{Ph}_{\mathrm{m}}\right), 124.8(\mathrm{Th}), 124.3(\mathrm{Th}), 123.9$ $\left(\mathrm{NPh}_{\mathrm{p}}\right), 123.4\left(\mathrm{NPh}_{\mathrm{o}}\right), 120.3(\mathrm{Th}), 35.2\left(\mathrm{CMe}_{3}\right), 31.4\left(\mathrm{CMe}_{3}\right), 22.8(o-\mathrm{Me}), 21.5(p-\mathrm{Me})$; UV-Vis $\left(\mathrm{CH}_{2} \mathrm{Cl}_{2}, 3.210^{-6}\right): \lambda_{\max }=309 \mathrm{~nm}(\varepsilon=26,700), 436 \mathrm{~nm}(\varepsilon=38,960)$; fluorescence $\left(\mathrm{CH}_{2} \mathrm{Cl}_{2}, 3.2\right.$ 
$\left.10^{-5} \mathrm{M}\right): \lambda_{\mathrm{em}, \max }=540 \mathrm{~nm}, \Phi=0.74\left(\lambda_{\mathrm{exc}}=436 \mathrm{~nm}\right) ; \mathrm{FAB}-\mathrm{MS} \mathrm{m} / z(\%): 595.2\left[\mathrm{M}^{+}\right](100) ;$

elemental analysis: calculated C 78.64, H 6.43, N 2.35; found C 78.00, H 6.54, N 2.46.

\section{References:}

(1) Sotgiu, G.; Zambianchi, M.; Barbarella, G.; Botta, C. Tetrahedron 2002, 58, 2245.

(2) (a) Tsuda, T.; Yazawa, T.; Watanabe, K.; Fujii, T.; Saegusa, T. J. Org. Chem. 1981, 46, 192-194. (b) Gambarotta, S.; Floriani, C.; Chiesi-Villa, A.; Guastini, C. J. Chem. Soc., Chem. Commun. 1983, 1156-1158. (c) Eriksson, H.; Håkansson, M. Organometallics 1997, 16, 4243-4244.

(3) Qin, Y.; Cheng, G.; Parab, K.; Achara, O.; Jäkle, F. Macromolecules 2004, 37, 71237131.

(4) Murov, S. L.; Carmichael, I.; Hug, G. L., Eds. Handbook of Photochemistry; 2nd ed.; Marcel Dekker Inc.: New York, 1993.

(5) Gans, P.; Sabatini, A.; A. Vacca Talanta 1996, 43, 1739-1753.

(6) Gaussian 03, Revision C.02, M. J. Frisch, G. W. Trucks, H. B. Schlegel, G. E. Scuseria, M. A. Robb, J. R. Cheeseman, J. A. Montgomery, Jr., T. Vreven, K. N. Kudin, J. C. Burant, J. M. Millam, S. S. Iyengar, J. Tomasi, V. Barone, B. Mennucci, M. Cossi, G. Scalmani, N. Rega, G. A. Petersson, H. Nakatsuji, M. Hada, M. Ehara, K. Toyota, R. Fukuda, J. Hasegawa, M. Ishida, T. Nakajima, Y. Honda, O. Kitao, H. Nakai, M. Klene, X. Li, J. E. Knox, H. P. Hratchian, J. B. Cross, C. Adamo, J. Jaramillo, R. Gomperts, R. E. Stratmann, O. Yazyev, A. J. Austin, R. Cammi, C. Pomelli, J. W. Ochterski, P. Y. Ayala, K. Morokuma, G. A. Voth, P. Salvador, J. J. Dannenberg, V. G. Zakrzewski, S. Dapprich, A. D. Daniels, M. C. Strain, O. Farkas, D. K. Malick, A. D. Rabuck, K. Raghavachari, J. B. Foresman, J. V. Ortiz, Q. Cui, A. G. Baboul, S. Clifford, J. Cioslowski, B. B. Stefanov, G. Liu, A. Liashenko, P. Piskorz, I. Komaromi, R. L. Martin, D. J. Fox, T. Keith, M. A. Al-Laham, C. Y. Peng, A. Nanayakkara, M. Challacombe, P. M. W. Gill, B. Johnson, W. Chen, M. W. Wong, C. Gonzalez, and J. A. Pople, Gaussian, Inc., Wallingford CT, 2004. 
Figure S1. TGA plots of polymers PSBMesBT, PSBMesHBT, and PSBMesNBT.

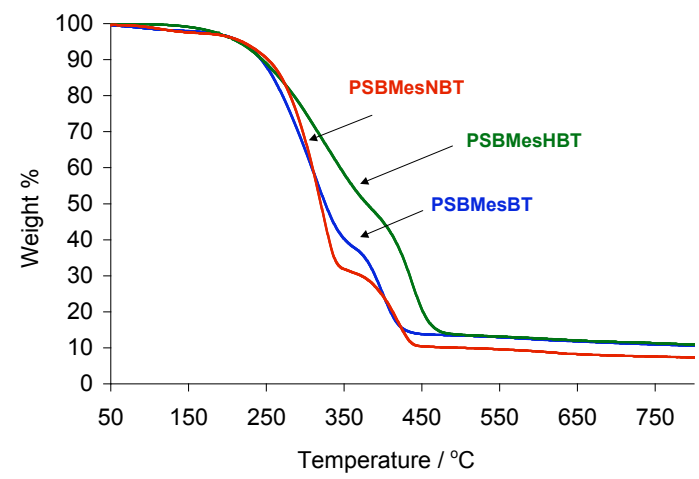

Figure S2. (a) Comparison of the absorption spectra of PSBMesHBT, PSBMesNBT, and the respective random copolymer in $\mathrm{CH}_{2} \mathrm{Cl}_{2}$ solution.

(b) Comparison of the emission spectra of PSBMesHBT, PSBMesNBT, and the respective random copolymer in $\mathrm{CH}_{2} \mathrm{Cl}_{2}$ solution (excitation at $\lambda_{\max }$ ).

a)

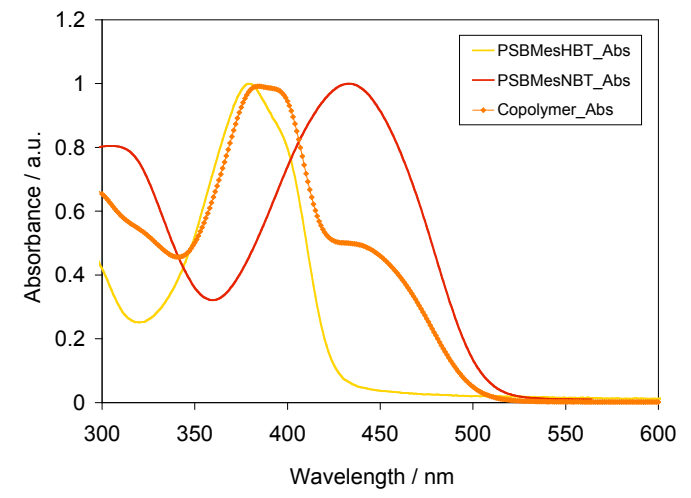

b)

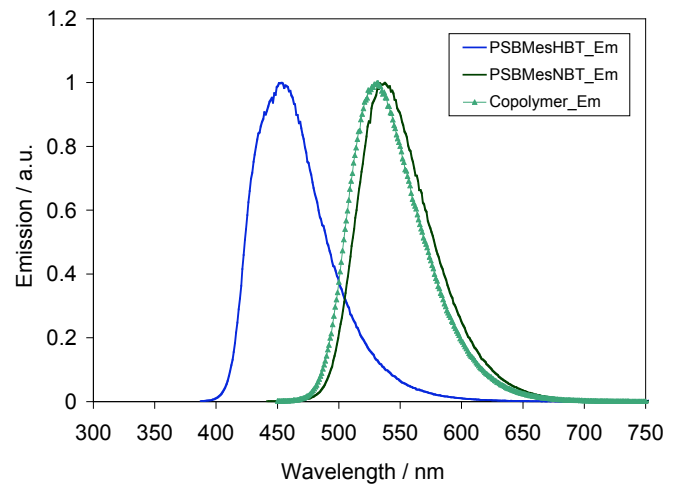

Figure S3. Determination of the fluoride binding constants.

Spectral titration data for MBMesHBT $\left(3.2310^{-5} \mathrm{M}\right.$ solution in THF) were obtained using a 4.36 $10^{-4} \mathrm{M}$ solution of $\mathrm{Bu}_{4} \mathrm{NF}$ as the titrant (20 titration points between 0 and 3 molar equivalents of fluoride). Refinement was performed using the Hyperquad ${ }^{\mathrm{TM}}$ program suite with 130 different wavelengths between 260 and $420 \mathrm{~nm}$. A model for a single binding event was used, where the binding constant $\mathrm{K}=\left[\mathrm{MBMesHBT}^{*} \mathrm{~F}^{-}\right] /\left([\mathrm{MBMesHBT}]\left[\mathrm{F}^{-}\right]\right)$was refined. For MBMesHBT $\operatorname{lgK}$ refined to $7.38 \pm 0.03$. Spectral titration data for PSBMesHBT $\left(1.7710^{-5} \mathrm{M}\right.$ repeat units in THF) were obtained using a $3.2010^{-4} \mathrm{M}$ solution of $\mathrm{Bu}_{4} \mathrm{NF}$ as the titrant (20 titration points between 0 to 4 molar equivalents of fluoride). The titration plots are shown in the manuscript. Refinement was performed using the Hyperquad ${ }^{\mathrm{TM}}$ program suite with 130 different wavelengths between 260 and $420 \mathrm{~nm}$. The binding constant was estimated assuming independent, non-communicating binding sites on the polymer. For PSBMesHBT $\operatorname{lgK}$ refined to $6.86 \pm 0.04$. 

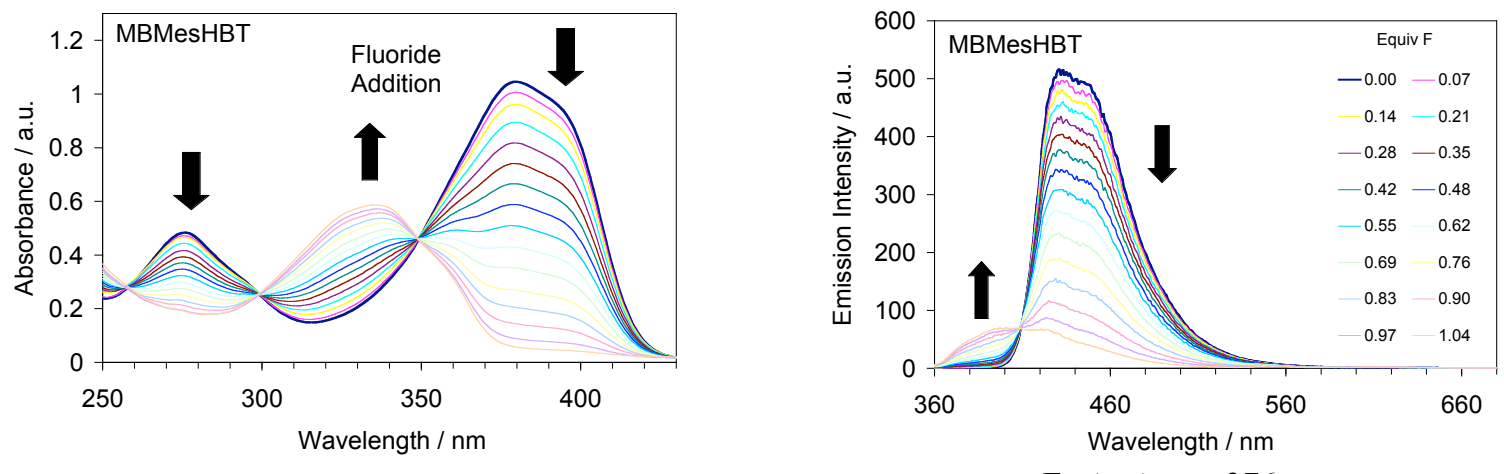

Excitation at $276 \mathrm{~nm}$

Spectral titration data for MBMesNBT (3.21 $10^{-5} \mathrm{M}$ solution in THF) were obtained using a 3.2 $10^{-4} \mathrm{M}$ solution of $\mathrm{Bu}_{4} \mathrm{NF}$ as the titrant (23 titration points between 0 and 3 molar equivalents of fluoride). Refinement was performed using the Hyperquad ${ }^{\mathrm{TM}}$ program suite with 200 different wavelengths between 265 and $495 \mathrm{~nm}$. A model for a single binding event was used, where the binding constant $\mathrm{K}=\left[\mathrm{MBMesNBT}^{*} \mathrm{~F}^{-}\right] /\left([\mathrm{MBMesNBT}]\left[\mathrm{F}^{-}\right]\right)$was refined. For MBMesNBT $\operatorname{lgK}$ refined to $7.60 \pm 0.03$. Spectral titration data for PSBMesNBT $\left(2.8910^{-5} \mathrm{M}\right.$ repeat units in THF) were obtained using a $3.2010^{-4} \mathrm{M}$ solution of $\mathrm{Bu}_{4} \mathrm{NF}$ as the titrant (22 titration points between 0 to 3 molar equivalents of fluoride). The titration plots are shown in the manuscript. Refinement was performed using the Hyperquad ${ }^{\mathrm{TM}}$ program suite with 200 different wavelengths between 266 and $491 \mathrm{~nm}$. The binding constant was estimated assuming independent, non-communicating binding sites on the polymer. For PSBMesNBT $\operatorname{lgK}$ refined to $6.94 \pm 0.04$.
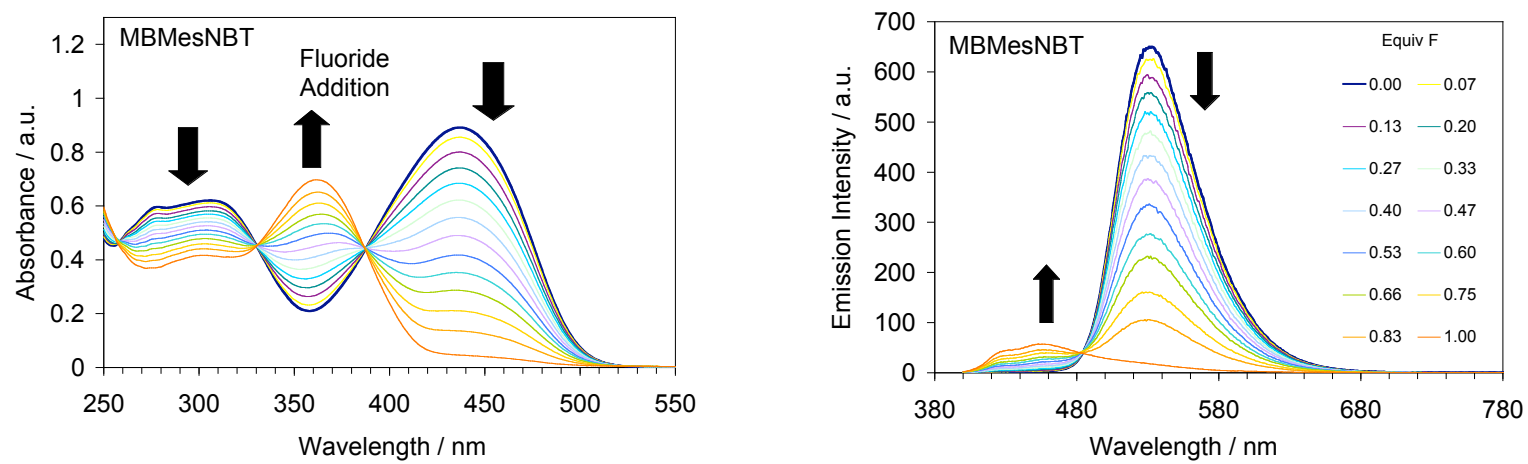

Excitation at $306 \mathrm{~nm}$

Figure S4. Comparison of the Stern-Volmer plots for the titration of MBMesHBT and PSBMesHBT, respectively, with $\mathrm{Bu}_{4} \mathrm{NF}$ in THF. To account for tight binding effects, the concentration of free fluoride as determined from refinement of the absorption data was used.

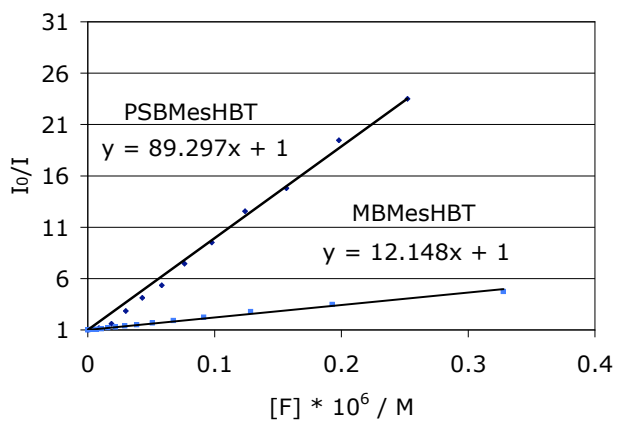


Table S1. Calculated orbital energies (eV) for PhBMesMeBT, PhBMesNBT, and their respective fluoride complexes from DFT (B3LYP) calculations.

\begin{tabular}{|c|c|c|c|c|}
\hline Compound & PhBMesMeBT & [PhBMesMeBT]F ${ }^{-}$ & PhBMesNBT & {$[$PhBMesNBT]F } \\
\hline \multirow[b]{2}{*}{ LUMO+2 } & 0.000 & 3.320 & 0.463 & 1.469 \\
\hline & $(\mathrm{Ph})$ & (Ph \& Mes) & $\left(\mathrm{Ph}_{2}\right.$ on $\left.\left.\mathrm{N}\right)\right)$ & $\left(\mathrm{NPh}_{2}\right)$ \\
\hline \multirow[b]{2}{*}{ LUMO+1 } & -0.762 & 3.156 & 0.844 & 1.361 \\
\hline & (bt-BPh) & (Th) & (bt-BPh) & $\left(\mathbf{N P h}_{2}\right)$ \\
\hline \multirow{2}{*}{ LUMO } & -1.986 & 1.687 & -1.959 & 1.252 \\
\hline & (bt-BPh) & (bt) & (bt-B) & (bt-NPh) \\
\hline \multirow[b]{2}{*}{ HOMO } & -5.497 & -2.095 & -4.952 & -2.286 \\
\hline & $(b t)$ & (bt) & $\left(\mathbf{P h}_{2} \mathrm{~N}-\mathrm{bt}\right)$ & (bt) \\
\hline \multirow[b]{2}{*}{ HOMO-1 } & -5.905 & -2.585 & -5.850 & -2.667 \\
\hline & (Mes) & (Mes) & (Mes) & (Mes) \\
\hline \multirow[b]{2}{*}{ HOMO-2 } & -6.068 & -2.857 & -6.014 & -2.939 \\
\hline & (Mes) & (Mes) & $\left(b t\right.$, Mes \& $\mathrm{NPh}_{2}$ ) & (Mes) \\
\hline
\end{tabular}

$\mathrm{bt}=2,2$-bithiophene; the orbitals that are involved in the calculated low energy transitions are marked in red and blue.

Table S2. Extended list of calculated electronic transitions for PhBMesMeBT, PhBMesNBT, and their respective fluoride complexes from TD-DFT (B3LYP) calculations.

\begin{tabular}{|c|c|c|c|c|}
\hline Compound & Transition & MO contributions & $\begin{array}{l}\text { Energy gap } \\
\mathrm{eV}(\mathrm{nm})\end{array}$ & Oscillator strength / f \\
\hline \multirow[t]{3}{*}{ PhBMesMeBT } & $\mathbf{S}_{\mathbf{0}} \rightarrow \mathrm{S}_{1}$ & $\begin{array}{l}\text { HOMO-1 } \rightarrow \text { LUMO } \\
\text { HOMO } \rightarrow \text { LUMO }\end{array}$ & $3.19(388)$ & 0.3452 \\
\hline & $\mathrm{S}_{0} \rightarrow \mathrm{S}_{2}$ & $\begin{array}{l}\text { HOMO-1 } \rightarrow \text { LUMO } \\
\text { HOMO } \rightarrow \text { LUMO }\end{array}$ & $3.27(379)$ & 0.4387 \\
\hline & $\mathrm{S}_{0} \rightarrow \mathrm{S}_{3}$ & HOMO-2 $\rightarrow$ LUMO & $3.47(357)$ & 0.0031 \\
\hline \multirow[t]{3}{*}{ [PhBMesMeBT]F- $^{-}$} & $\mathrm{S}_{0} \rightarrow \mathrm{S}_{1}$ & HOMO $\rightarrow$ LUMO & $3.53(351)$ & 0.6596 \\
\hline & $\mathrm{S}_{0} \rightarrow \mathrm{S}_{2}$ & HOMO-1 $\rightarrow$ LUMO & $3.84(323)$ & 0.0399 \\
\hline & $\mathrm{S}_{0} \rightarrow \mathrm{S}_{3}$ & $\begin{array}{l}\text { HOMO- } 3 \rightarrow \text { LUMO } \\
\text { HOMO-4 } \rightarrow \text { LUMO }\end{array}$ & $4.14(300)$ & 0.0042 \\
\hline \multirow[t]{3}{*}{ PhBMesNBT } & $S_{0} \rightarrow S_{1}$ & HOMO $\rightarrow$ LUMO & $2.65(468)$ & 1.2544 \\
\hline & $\mathrm{S}_{0} \rightarrow \mathrm{S}_{2}$ & HOMO- $1 \rightarrow$ LUMO & $3.22(384)$ & 0.0049 \\
\hline & $\mathrm{S}_{0} \rightarrow \mathrm{S}_{3}$ & $\begin{array}{l}\text { HOMO- } 3 \rightarrow \text { LUMO } \\
\text { HOMO-4 } \rightarrow \text { LUMO }\end{array}$ & $3.47(357)$ & 0.0015 \\
\hline \multirow[t]{3}{*}{${\text { PhBMesNBT }] F^{-}}^{-}$} & $\mathrm{S}_{0} \rightarrow \mathrm{S}_{1}$ & $\begin{array}{l}\text { HOMO } \rightarrow \text { LUMO } \\
\text { HOMO } \rightarrow \text { LUMO+1 }\end{array}$ & $3.22(386)$ & 0.3125 \\
\hline & $\mathbf{S}_{0} \rightarrow \mathbf{S}_{2}$ & $\begin{array}{l}\text { HOMO } \rightarrow \text { LUMO } \\
\text { HOMO } \rightarrow \text { LUMO+1 }\end{array}$ & $3.23(384)$ & 0.5713 \\
\hline & $\mathrm{S}_{0} \rightarrow \mathrm{S}_{3}$ & $\mathrm{HOMO} \rightarrow \mathrm{LUMO}+2$ & $3.33(372)$ & 0.0050 \\
\hline
\end{tabular}


Molecular Mechanics (MM+) calculations of a methyl-capped trimer were carried out using HyperChem 6.01 (Hypercube, Inc., Waterloo, Ontario, Canada). The minimization was allowed to proceed until the residual RMS force of the structure went below $0.05 \mathrm{kcal} / \mathrm{mol} / \AA$. The optimized structures of the trimer with all bithiophenes moieties pointing into the same direction and the respective fluoride complexes are shown. Other orientations where the position of one of the bithiophene groups was switched with that of the mesityl substituent led to considerably higher energies. Due to their large number, other possible stereoisomers were not studied.

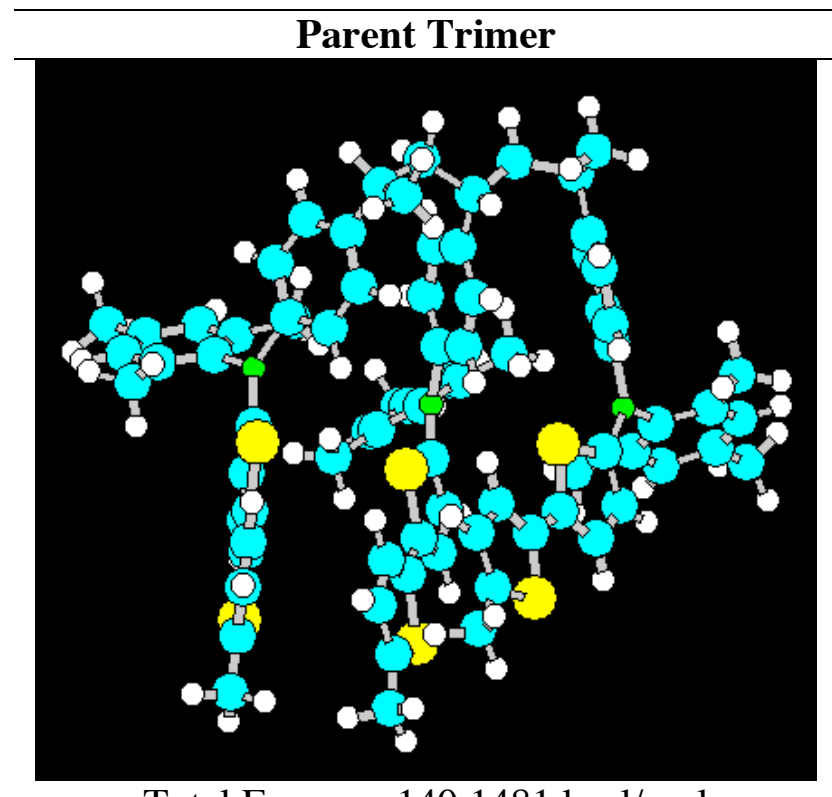

Total Energy $=140.1481 \mathrm{kcal} / \mathrm{mol}$

\section{Terminal Fluoride}

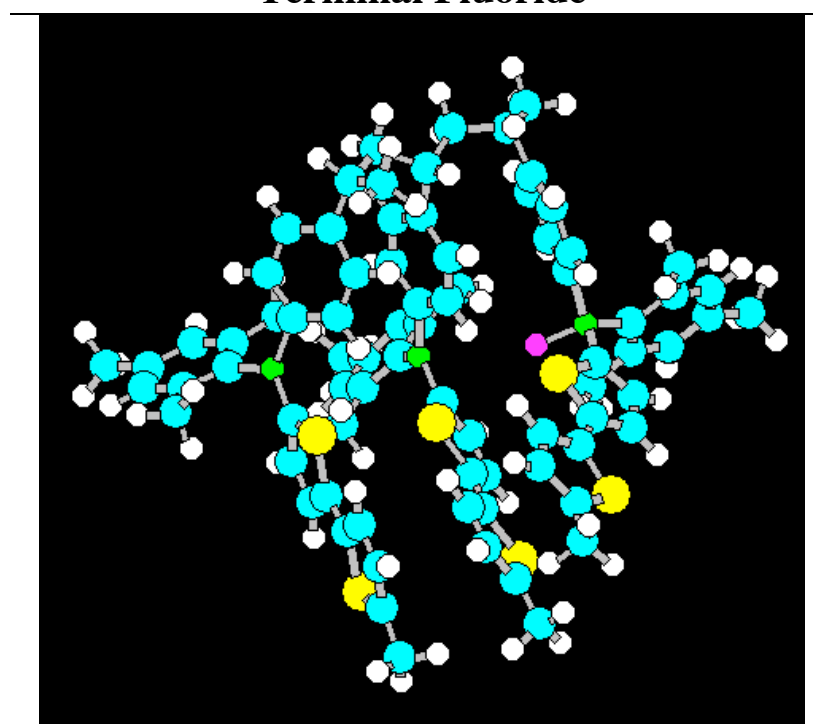

Total Energy $=147.5452 \mathrm{kcal} / \mathrm{mol}$
Bridging Fluoride

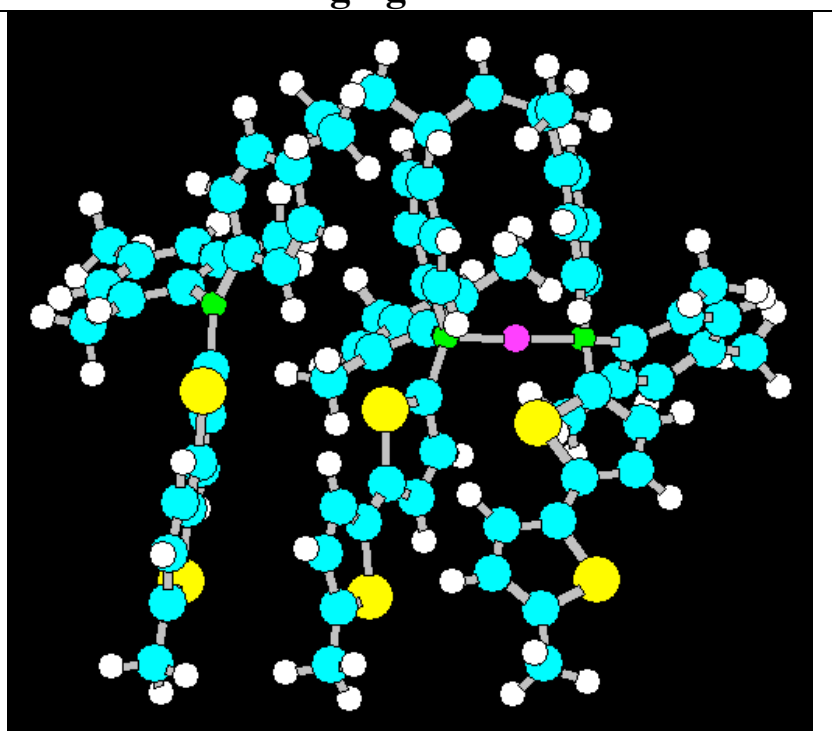

Total Energy $=163.0683 \mathrm{kcal} / \mathrm{mol}$

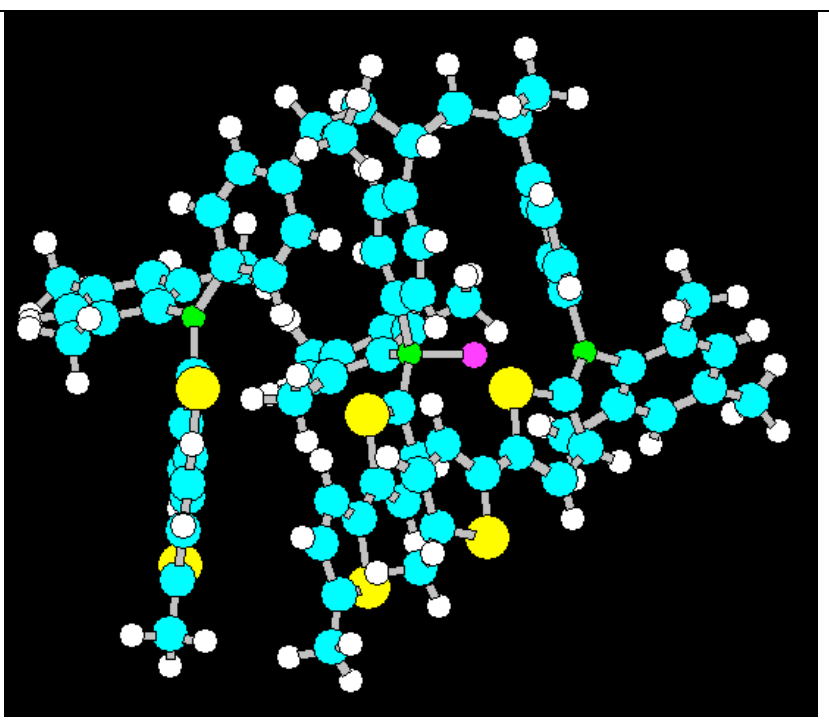

Total Energy $=149.3913 \mathrm{kcal} / \mathrm{mol}$ 